\title{
Promoting Dendrimer Self-Assembly Enhances Covalent Layer-by- Layer Encapsulation of Pancreatic Islets
}

\author{
KM Gattás-Asfura ${ }^{1}$, NJ Abuid ${ }^{1}$, I Labrada ${ }^{1}$, and CL Stabler ${ }^{1-3^{*}}$
}

${ }^{1} \mathrm{~J}$. Crayton Pruitt Family Department of Biomedical Engineering, University of Florida, Gainesville, FL, USA

${ }^{2}$ Interdisciplinary Program in Biomedical Sciences, University of Florida, Gainesville, FL, USA

${ }^{3}$ University of Florida Diabetes Institute, Gainesville, FL, USA

\section{SUPPLEMENTARY FIGURES}

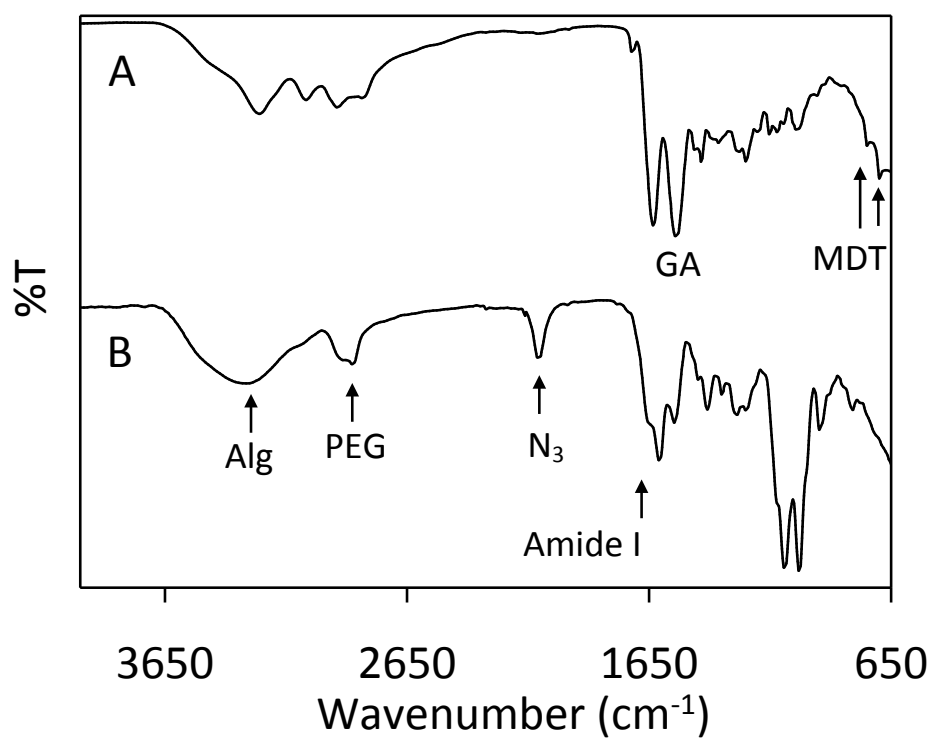

Figure S1. ATR-FTIR spectra for (A) PAMAM-MDT/GA/SIL (top) and (B) azido-functionalized hyperbranched alginate (bottom). 


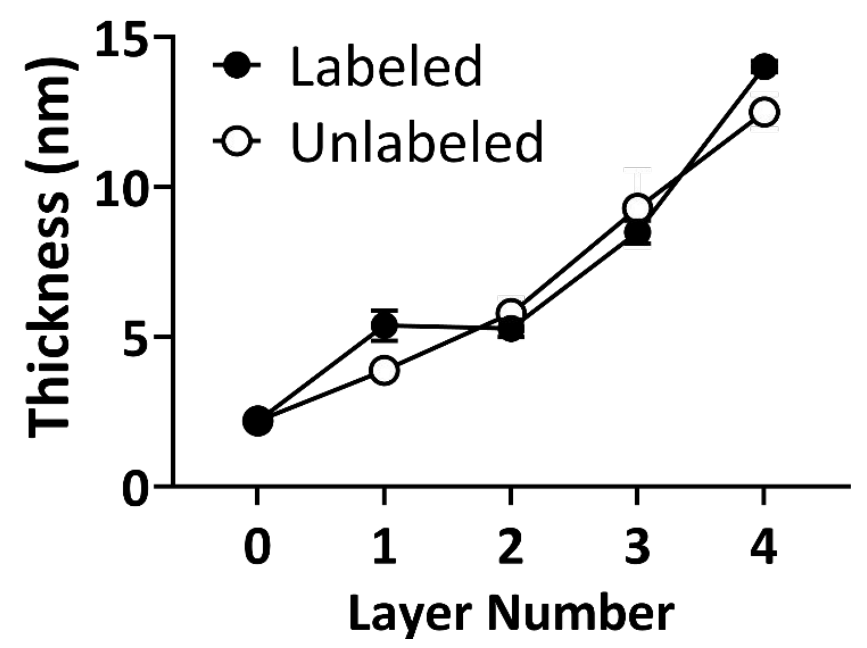

Figure S2. Ellipsometry measurement of layer formation onto idealized planar surfaces with alternating solutions of PAMAM and hyperbranched alginate, with or without fluorescein label, designed as Labeled (filled circles) or Unlabeled (open circles), respectively. Two way ANOVA noted no significant effect of the presence of the fluorescein label on layer formation $(p=0.0932)$. 


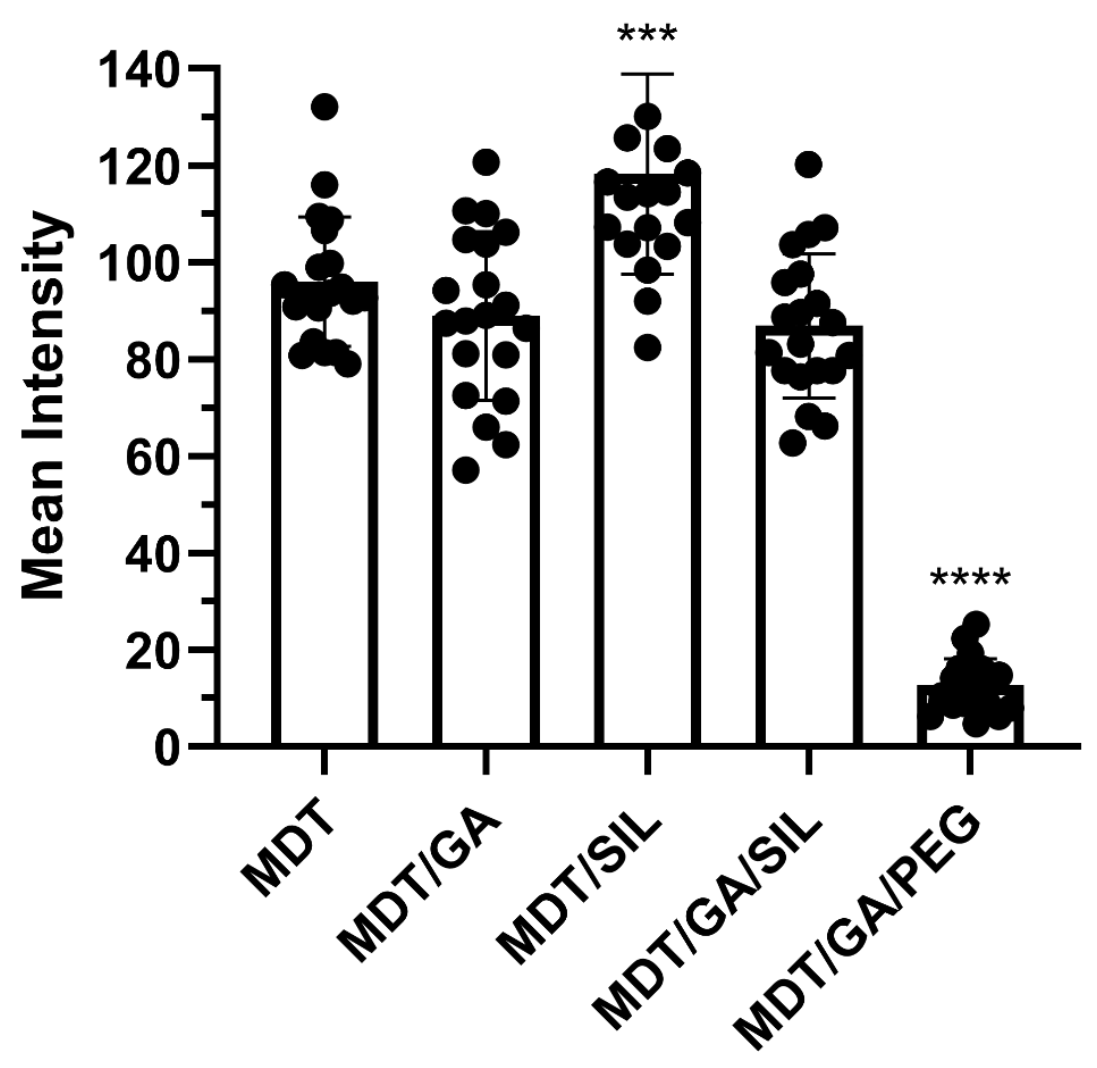

PAMAM Functionalization

Figure S3. SIL functionalization enhances deposition of polymer coatings. Quantification of the deposition of Alg-HyPEG-N3 (green) 1-day after coating islets with 4 alternating layers containing different PAMAM derivatives. $* * * * p<0.0001$ from all other groups; $* * * p<0.001$ from all other groups; one-way ANOVA with Tukey post-hoc. 

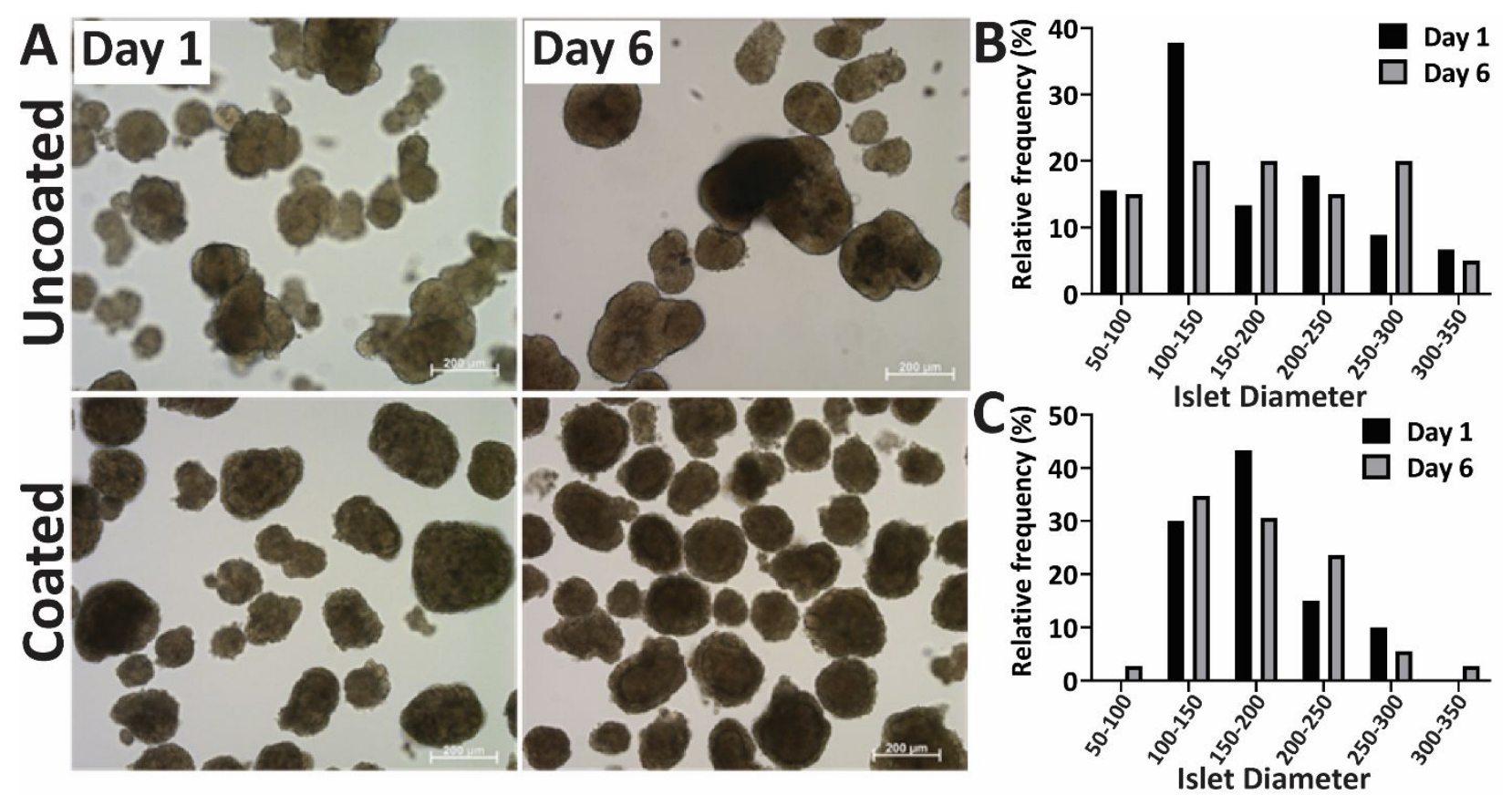

Figure S4. Characterization of islet morphology and size for cLbL coated and uncoated islets over culture time. A) Light microscopy images of uncoated (top row) and cLbL coated (bottom row) islets on days 1 and 6 post-coating. B-C) Quantification of islet size, binned per islet diameter, for uncoated $(\mathrm{B})$ and coated $(\mathrm{C})$ islets on days 1 and 6 post-coating. 


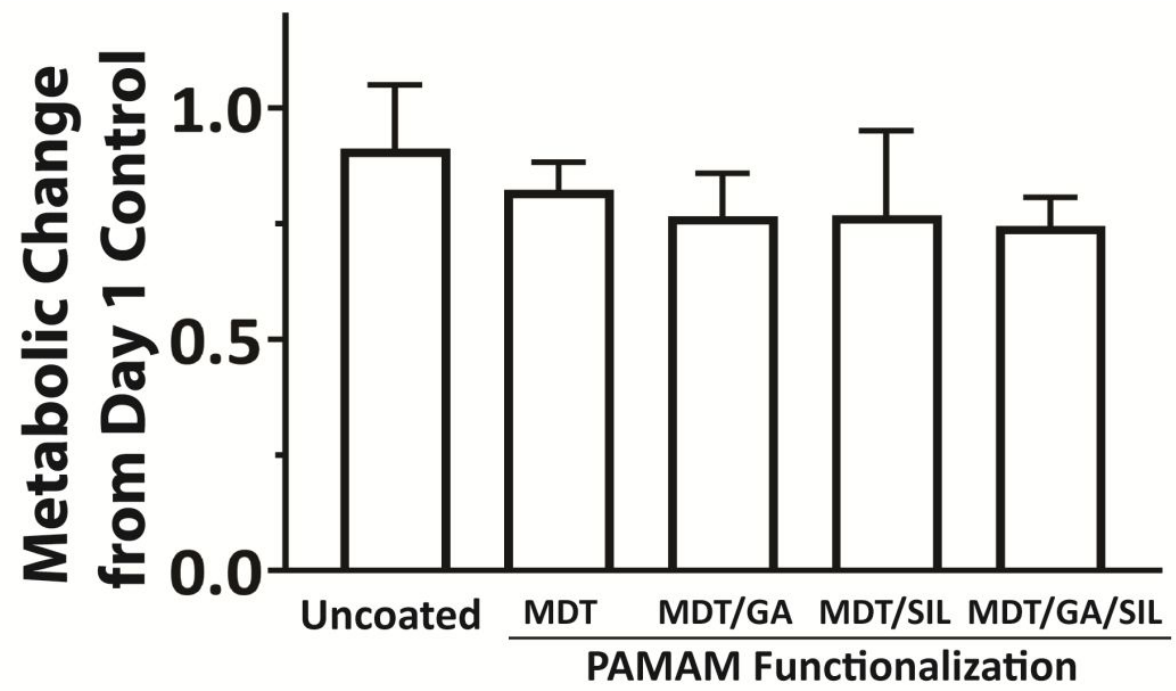

Figure S5. PAMAM-based cLbL coatings impart minimal changes in islet metabolic activity. Metabolic activity, per MTT assay, for uncoated and 4-layer coated islets as a function of PAMAM functionalization. Measurements were made 5 day after coating and normalized to day 1 control readings. No significant effect of coating approach on metabolic activity was measured (ANOVA with Tukey post-hoc)

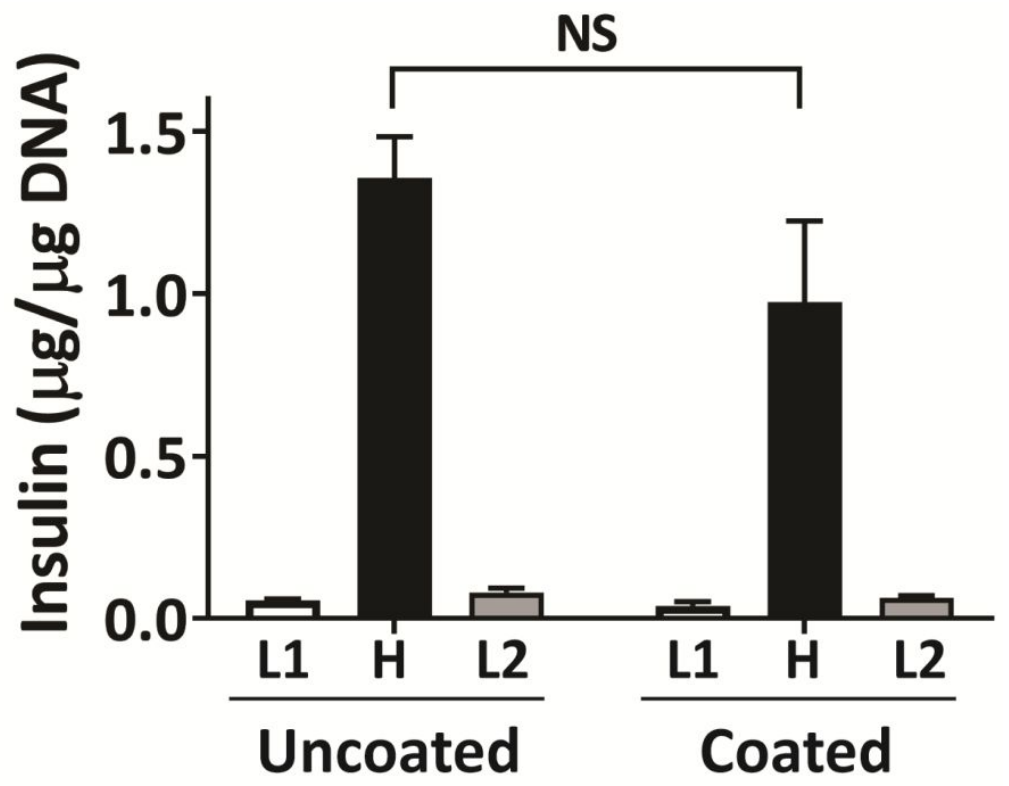

Figure S6. PAMAM-based cLbL coatings impart minimal changes in islet function. Glucose stimulated insulin release for uncoated control and 4-layer coated islets. 


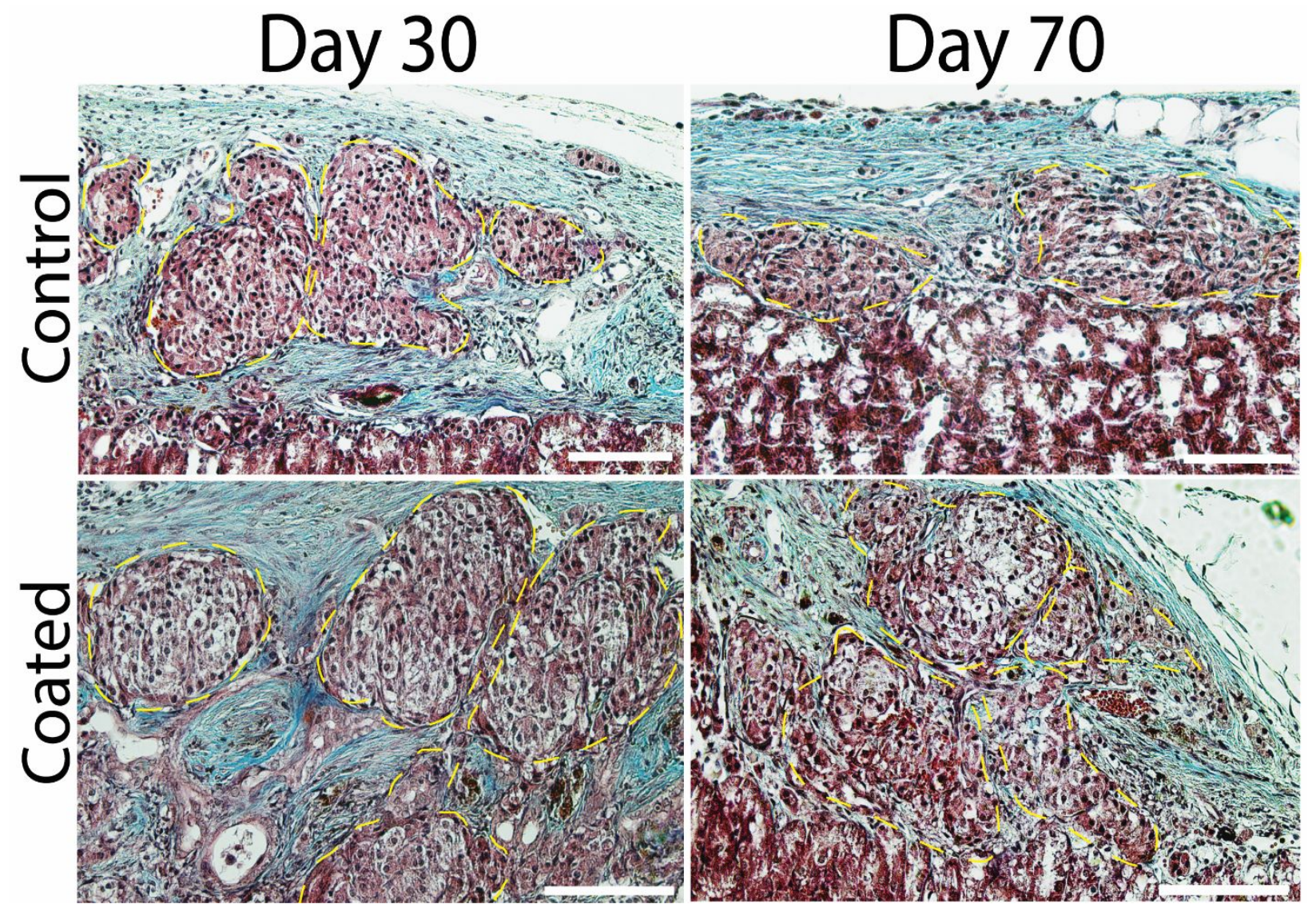

Figure S7. Histological analysis of explanted grafts after 30 and 70 days post-transplantation. A-D) Masson's trichrome stain of control (top row) and coated (bottom row) islets after 30 (left column) or 70 days (right column) post-transplant. Scale bar $=100 \mu \mathrm{M}$. 